\title{
Investigating a galactomannan gel obtained from Cassia grandis seeds as immobilizing matrix for Cramoll lectin
}

\author{
Priscilla B.S. Albuquerque ${ }^{a}$, Caroline S. Silva ${ }^{b}$, Paulo A.G. Soares ${ }^{b}$, Wilson Barros Jr. ${ }^{c}$, \\ Maria T.S. Correia ${ }^{\mathrm{b}}$, Luana C.B.B. Coelho ${ }^{\mathrm{b}}$, José A. Teixeira ${ }^{\mathrm{d}}$, \\ Maria G. Carneiro-da-Cunha ${ }^{\mathrm{a}, \mathrm{b}, *}$ \\ ${ }^{a}$ Laboratório de Imunopatologia Keizo Asami, Universidade Federal de Pernambuco, Campus Universitário, s/n, Cidade Universitária, CEP: 50670-901 \\ Recife, PE, Brazil \\ b Departamento de Bioquímica, Universidade Federal de Pernambuco (UFPE), Av. Prof. Moraes Rego, s/n, Cidade Universitária, CEP: 50670-420 Recife, PE, \\ Brazil \\ ${ }^{\mathrm{c}}$ Departamento de Física, Universidade Federal de Pernambuco, Avenida prof. Luiz Freire, s/n, CEP: 50670-901 Recife, PE, Brazil \\ d IBB-Institute for Biotechnology and Bioengineering, Centre of Biological Engineering, Universidade do Minho, Campus de Gualtar, 4710-057 Braga, \\ Portugal
}

\section{A R T I C L E I N F O}

\section{Article history}

Received 17 October 2015

Received in revised form 27 January 2016

Accepted 28 January 2016

Available online 1 February 2016

\section{Keywords:}

Cassia grandis

Cramoll

Galactomannan

Rheology

Stability

\begin{abstract}
A B S T R A C T
Characterization, with emphasis on the rheological properties, of Cassia grandis seeds galactomannan gel containing immobilized Cramoll 1-4 is presented. The gels, with and without immobilized Cramoll 1-4, were evaluated along time by rheometry, $\mathrm{pH}$, color, microbial contamination and lectin hemagglutinating activity (HA). Rheological determinations confirmed the gels to be very stable up to 30 days with variations occurring after this period. Rheological data also showed that the gel/Cramoll 1-4 immobilizing matrix loses its elastic modulus substantially after 60 days. Both gels presented no microbial contamination as well as a pH close to neutral. Colorimetric parameters demonstrated the gels transparency with occasional yellowness. The opacity of the galactomannan gel did not change significantly along the study; the same did not occur for the gel with immobilized Cramoll 1-4 as a statistically significant reduction of its opacity was observed. In what concerns immobilized Cramoll 1-4 HA, up to 90\% of its initial HA was maintained after 20 days, with a decrease to $60 \%$ after 60 days. These results combined with the thickening and stabilizing characteristics of the galactomannan gel make this gel a promising immobilizing matrix for Cramoll 1-4 that can be further exploited for clinical and cosmetic applications.
\end{abstract}

(c) 2016 Elsevier B.V. All rights reserved.

\section{Introduction}

Polysaccharides isolated from natural sources have been attracting much attention in the biochemistry and pharmacology fields [1] thanks to their bioactivity, biocompatibility, low cost and availability [2]. Galactomannans extracted from the endosperm of numerous plants (particularly Leguminosae) are polysaccharides widespread in nature. Their basic structure is composed by a central core of $\alpha(1 \rightarrow 4)$-linked D-mannopyranose to which $\beta(1 \rightarrow 6)$ linked $\alpha$-D-galactopyranosyl units are attached. High molecular weight, non-ionic character [3], and water solubility allow these

\footnotetext{
* Corresponding author. Fax: +55 8121268576.

E-mail addresses: mgcc1954@gmail.com, mgcc@pq.cnpq.br (M.G. Carneiro-da-Cunha)
}

systems to forming highly viscous and stable solutions at low concentrations [4].

Galactomannans are used in food, pharmaceutical, biomedical, cosmetic, textile and paper industries. Many studies have reported the potential use of galactomannans as thickeners [5], part of mixed systems such as hydrogels [6], emulsion stabilizers in the preparation of films [7] and edible films themselves with enhanced barrier as well as mechanical properties that extend and improve food shelf-life $[8,9]$. It is also important to highlight the application of galactomannans in the cosmetic industry [10] and as matrix for immobilization of certain biomolecules [11-14].

Galactomannan solutions exhibit non-Newtonian behavior and their rheological properties are also connected to their concentration [15], molar mass [5], and the mannose/galactose (M/G) ratio [16]. In order to guarantee the application efficacy of galactomannans in cosmetic and pharmaceutical fields, a full rheological characterization is usually necessary [2] as is the case of the Cross 
model [17] that has been used successfully to describe the shearthinning properties of galactomannans $[18,19]$.

Albuquerque et al. [20] have worked at an extensive rheological characterization of the galactomannan extracted from the seeds of Cassia grandis, a typical Brazilian tree, and observed that it presents fluid behavior, with Newtonian plateau followed by shearthinning zones up to $1.5 \%(\mathrm{w} / \mathrm{v})$ concentrations; above this value, the galactomannan behavior can be considered as that of a polymer solution of entangled strands. At $2.0 \%$ concentration, rheological data indicate that the system has reached a gel-like state which, in rheological terms, is one where the elastic modulus $\mathrm{G}^{\prime}$ is larger than the viscous modulus $G^{\prime \prime}$ and their dependence on oscillatory test frequency is low. The stress-strain study allowed a more accurate evaluation of this fluid-gel transition indicating that galactomannan concentrations higher than $1.7 \%(\mathrm{w} / \mathrm{v})$ could be suitable for a range of applications including immobilizing matrix for certain biomolecules.

Lectins constitute a protein heterogeneous group of nonimmune origin, containing two or more binding sites to mono or oligosaccharides [21]. The lectin extracted from Cratylia mollis seeds, also known as Cramoll, is a biomolecule with different molecular forms (Cramoll 1, Cramoll 2, Cramoll 3 and Cramoll 4) that has been well studied by structural analysis and employed in several biotechnological applications. In addition, its specificity is correlated with the so studied Concanavalin A lectin. Cramoll 1, Cramoll 2, and Cramoll 4 specifically bind to glucose or mannose, while Cramoll 3 is a galactose-specific glycoprotein [22,23]. Preparations containing isoforms 1 and 4 (Cramoll 1-4) were reported as presenting promising activities: antitumor [24]. anti-parasitic $[25,26]$, anti-inflammatory $[27,28]$ and mitogenic $[29,30]$. Cramoll $1-4$ is also able to bind, isolate, and characterize human plasma glycoproteins [31] and to act as a wound healing agent [32].

The technological development of novel forms for lectin immobilization has been a promising strategy to increase the penetration of drugs through the skin. The galactomannan gel obtained from the seeds of $C$. grandis has advantageous features for topical applications, especially due to the intimate contact made with the stratum corneum. Regarding these promising characteristics, developing an immobilizing matrix for Cramoll 1-4 based on the galactomannan gel would be a very important first step towards an improved drug delivery system for topical applications. By utilizing a series of tests ( $\mathrm{pH}$, colorimetry, rheometry, microbial contamination, and biological activity), the performance of the galactomannan gel at $1.7 \%$ $(\mathrm{w} / \mathrm{v})$ concentration, to work as a matrix for immobilizing Cramoll 1-4 was evaluated in this work. The above mentioned tests were conducted on specific days along a total experimental interval of 90 days, during which gel matrix samples were stored under controlled temperature and relative humidity conditions. Samples of the original gel, with no Cramoll 1-4, submitted to the same conditions of the immobilizing matrix system, were used for controlled comparison.

\section{Materials and methods}

The pods of $C$. grandis were collected at the rural zone of Pernambuco State, in the city of Angelim (Brazil), in July 2011. Ethanol $99.8 \%$, acetone PA, sodium chloride and phenol were obtained from Vetec Fine Chemicals Ltd. (Brazil). All other chemicals were of analytical grade.

\subsection{Extraction of the galactomannan from $\mathrm{C}$. grandis seeds and gel preparation}

The galactomannan from $C$. grandis seeds was obtained according to Albuquerque et al. [20]. Briefly, C. grandis pods were immersed in distilled water at $25^{\circ} \mathrm{C}$ for $18 \mathrm{~h}$ and then separated in a half part, revealing the seeds trapped in a black and stick mass. The seeds were removed and placed dried until reaching a constant weight. The dry seeds were boiled in distilled water 1:5 (w/v) at $100^{\circ} \mathrm{C}$ for $1 \mathrm{~h}$ for enzyme inactivation and maintained in water by $18 \mathrm{~h}$ at $25^{\circ} \mathrm{C}$ to facilitate removal of the hull. After this period, the hull was removed and the residual was triturated in a blender with $0.1 \mathrm{M} \mathrm{NaCl} 5 \%(\mathrm{w} / \mathrm{v})$ at $25^{\circ} \mathrm{C}$, filtered through a voil tissue and after using a screen printing cloth, and precipitated with $46 \%$ ethanol $1: 3(\mathrm{v} / \mathrm{v})$ for $18 \mathrm{~h}$. The white precipitate obtained was washed with $100 \%$ ethanol 1:3 (w/v) for $30 \mathrm{~min}$ and two times with acetone PA $1: 3(\mathrm{w} / \mathrm{v})$ for $30 \mathrm{~min}$, been filtered on screen printing cloth between each washing. The precipitate was dried until constant weight, pulverized and finally called galactomannan.

The galactomannan was dissolved in distilled water at $1.7 \%$ $(\mathrm{w} / \mathrm{v})$ under magnetic stirring $(500 \mathrm{rpm})$ for $12 \mathrm{~h}$ at room temperature $\left(25^{\circ} \mathrm{C}\right)$. The obtained gel was stored at $4{ }^{\circ} \mathrm{C}$ and $80 \%$ relative humidity, been called pure galactomannan gel.

\subsection{Extraction of the lectin from $\mathrm{C}$. mollis seeds and immobilization on the galactomannan gel}

Cramoll, containing its isoforms 1 and 4 (Cramoll 1-4) was obtained according to Correia and Coelho [22]. Briefly, the seeds were dried at $25^{\circ} \mathrm{C}$ and crushed to obtain a flour, which was dissolved in $0.15 \mathrm{M} \mathrm{NaCl} 10 \%(\mathrm{w} / \mathrm{v})$, under magnetic stirring (500 rpm) for $18 \mathrm{~h}$ at $4{ }^{\circ} \mathrm{C}$. This saline extract was fractionated with ammonium sulphate (0-40\% and 40-60\%). The supernatant of the $40-60 \%$ fraction was dialysed and purified by affinity chromatography on Sephadex G-75 column.

Cramoll 1-4 was immobilized by entrapment on pure galactomannan gel as follows: $100 \mu \mathrm{g} / \mathrm{ml}$ of Cramoll $1-4$ were added to the pure galactomannan gel under magnetic stirring $(500 \mathrm{rpm})$ for $1 \mathrm{~h}$ at room temperature $\left(25^{\circ} \mathrm{C}\right)$. The gel with immobilized Cramoll $1-4$, called gel/Cramoll 1-4 immobilizing matrix, was stored at $4{ }^{\circ} \mathrm{C}$ and $80 \%$ relative humidity.

The activity of Cramoll 1-4 free solution and Cramoll 1-4 immobilized on the gel matrix was evaluated by hemagglutinating activity (HA) assay according to the methodology described by Correia and Coelho [22]. using a suspension [2.5\% (v/v)] of rabbit erythrocytes treated with glutaraldehyde [33]. All HA results were expressed as log.

\subsection{Stability of the pure galactomannan gel and the gel with immobilized Cramoll 1-4}

The stability of the pure galactomannan gel and the gel/Cramoll 1-4 immobilizing matrix was evaluated by $\mathrm{pH}$, colorimetry, and rheology for 90 days, been examined on the following days: $1,5,10$, $20,30,60$ and 90 . All gel/Cramoll 1-4 immobilizing matrix samples and pure gel samples were prepared following systematic methods and controlled conditions where distinct samples, from the same initially prepared batch, were tested along different periods of storage.

The $\mathrm{pH}$ determination was conducted on a digital $\mathrm{pH}$ meter where two buffer solutions with $\mathrm{pH} 4.00$ and 7.00 were employed for calibration. Three measurements of $\mathrm{pH}$ for each sample were performed for statistical analysis and their average was used. The color quantification was performed by a digital colorimeter (Konica Minolta, model CHROMA METER CR-410, Osaka, Japan) calibrated at illuminant $C$ with a white standard. The parameters determined were $L^{*}\left(L^{*}=0\right.$ [black] and $L^{*}=100$ [white]), $a^{*}\left(-a^{*}=\right.$ greenness and $+a^{*}=$ redness $)$ and $b^{*}\left(-b^{*}=\right.$ blueness and $+b^{*}=$ yellowness $)$. These parameters are the ones recommended by the International Commission on Illumination. The opacity $(Y)$ was calculated according to the Hunterlab color scale as the ratio between opacity of 
each sample on the black standard $(Y \mathrm{~b})$ and opacity of each sample on the white standard $(Y w)$. The results were expressed as a percentage: $Y(\%)=100(Y \mathrm{~b} / \mathrm{Yw})$. The analyses of mesophilic aerobes, yeasts and molds, lactic acid bacteria and psychrotrophs, to evaluate the microbiological contamination of the both gels, were carried according to the methodology described by the Compendium of Methods for the Microbiological Examination of Foods [34]. All measurements were performed in triplicate and the results expressed as mean \pm standard deviation.

The mechanical stability studies were conducted on a stress controlled rheometer (Anton Paar MCR 301) equipped with a temperature controller. A circular parallel plate cell with internal diameter (i.d.) $=25 \mathrm{~mm}$ and a height $(h)=1 \mathrm{~mm}$ gap between plates was employed in the experiment. All rheology experiments were conducted at $25^{\circ} \mathrm{C}$. In order to guarantee reproducibility, for every experiment it was run a newly prepared sample, obtained from the same batch.

Stress-strain tests, rotational flow, and oscillatory viscoelastic linear-response measurements have been performed. The rheometer was operated in the oscillatory mode to the stress-strain tests: for a fixed frequency $\omega=2 \mathrm{rad} / \mathrm{s}$, an externally applied shear stress varied from 1 to $300 \mathrm{~Pa}$ and the storage $\left(\mathrm{G}^{\prime}\right)$ and loss $\left(\mathrm{G}^{\prime \prime}\right)$ moduli were recorded. That allows the sample mechanical responses to cover from the reversible linear response regime up to the nonlinear region, where the sample structure collapses and flow starts. Each experimental point was recorded after an exposure period of $5 \mathrm{~s}$. The flow study was conducted in continuous steady state shear rotational mode by varying the shear-rate from 1 to $1000 \mathrm{~s}$ while acquiring 50 experimental points per run. The dynamic oscillatory mode study was conducted varying the applied torque/deformation angular frequency from 1 to $500 \mathrm{rad} / \mathrm{s}$. The storage $\left(G^{\prime}\right)$ and loss $\left(G^{\prime \prime}\right)$ modulus sample responses were recorded during frequency sweeps at $0.2 \%$ fixed strain amplitude, enough to guarantee linear reversible responses. Again, each experimental point was recorded after a $5 \mathrm{~s}$ exposure period.

\subsection{Statistical analysis}

The results were expressed as mean \pm standard deviation, submitted to ANOVA (one-way) and Tukey's multiple comparison test. The values were considered statistically significant if compared to the significance level of $p \leq 0.05$. All statistical and graphical analyses were carried out with the Statistica 8.0 program (SatSoft Inc., 2008, Tulsa, OK, USA).

\section{Results and discussion}

The galactomannan extracted from the seeds of $C$. grandis showed extraction yield of $36 \pm 8 \%$ and presented a mixture of $71.0 \%$ mannose and $29.0 \%$ galactose, identical to the reported by Albuquerque et al. [20]. No additional sugars were detected in the NMR spectra of this polysaccharide and the mannose/galactose ratio was 2.44:1. Cramoll 1-4 was efficiently extracted with the same chromatographic and electrophoretic profile reported by Correia and Coelho [22].

\subsection{Stability investigation}

\subsection{1. $p H$ value}

The ability of galactomannans to form viscous solutions at relatively low concentration is their greatest advantage [17]. Furthermore aqueous solutions of galactomannan are basically neutral [35] and their viscosity is insensitive to $\mathrm{pH}$ variations (a broad range from 1 to 10.5); nevertheless some degradation may occur under highly acidic or alkaline conditions $[7,35]$.
The $\mathrm{pH}$ stability assay resulted in values of $6.82 \pm 0.16$ for the pure galactomannan gel and $6.80 \pm 0.39$ for the gel/Cramoll $1-4$ immobilizing matrix. The measured values were based on averages where no statistically significant variations $(p \geq 0.05)$ over time were found. The viscosity and the $\mathrm{pH}$ stability of galactomannans depend on the initial preparation as well as the storage conditions. In our case, the close to neutral $\mathrm{pH}$ measured for both gels over time is considered optimum for most biotechnological applications.

\subsubsection{Color}

Color and appearance are important features for selection or acceptance of a product from food industry [36] to the cosmetics [37]. The galactomannan gel obtained from C. grandis seeds, with and without immobilized Cramoll 1-4, was characterized by instrumental colorimetry to ensure that color variations over storage time would not adversely affect the properties of product conservation.

The colorimetric $L^{*}, a^{*}$ and $b^{*}$ parameters of the pure galactomannan gel and the gel/Cramoll immobilizing matrix were expressed based on averages of the measured values, which did not showed statistically significant differences $(p>0.05)$ over time. The measured values for pure galactomannan gel were $L^{*}(91.74 \pm 1.32), a^{*}(-0.40 \pm 0.14)$ and $b^{*}(4.66 \pm 0.40)$, similar to those obtained for gel/Cramoll 1-4 immobilizing matrix: $L^{*}$ $(88.73 \pm 2.97), a^{*}(-0.36 \pm 0.18)$ and $b^{*}(5.00 \pm 0.54)$. Both the pure galactomannan gel and the gel/Cramoll 1-4 immobilizing matrix had a strong whiteness tendency, represented by the $L^{*}$ coordinate, a weak green color and special evidence for the yellowness appearance, respectively due to $a^{*}$ and $b^{*}$. These steady values indicated minimal loss of color and thus confirmed the preservation of the gel initial state over time. This emerges as an advantage for the natural products (extracted from seeds) when compared to those obtained by fruit puree, which have the natural tendency to browning [38]. In addition, the pure galactomannan gel did not present significant color variations as those constituted, for example, by banana pulp, which tend to vary from deep yellow to red due to chemical reactions such as Maillard [38].

Opacity indicates the capacity of biopolymers for acting as barrier to light; and also can be reported as a way to relate their higher or lower miscibility [39]. The pure galactomannan gel has a three-dimensional network with a natural and stable organization, which might occur due to the high degree of substitution of galactose units in the main chain of mannose. Statistically, the opacity was not significantly different during the storage period for the pure galactomannan gel $(10.49 \pm 1.14)$. In turn, the gel/Cramoll $1-4$ immobilizing matrix presented a statistically significant variation for the opacity value, with a decrease from $12.39 \pm 0.64$ on day 1 to $8.53 \pm 0.38$ on the 30 th day. A small increase occurred on the 60th day $(12.76 \pm 1.66)$ and another reduction on the 90th day, at the final of the experimental time $(9.83 \pm 1.29)$. These results suggest that opacity depends on the structure level of the galactomannan as well as the interactions with other biomolecules; furthermore the opacity loss as a function of structure integrity is also corroborated by the rheological results reported in Section 3.1.3, where the apparent viscosity is reduced for a long storage period.

Our results are in agreement with Soares et al. [6]. who developed a new hydrogel based upon optimized rheological characteristic of a mixture of $\kappa$-carrageenan and the galactomannan extracted from $C$. grandis seeds at the same concentration as that from our work. They also observed changes in opacity with the aging of the hydrogel, thus confirming the reorganization of the polysaccharide chains of the hydrogel that was reflected in a less opaque macroscopic sample.

\subsubsection{Rheological study}

Polysaccharide solutions are considered viscoelastic materials because they exhibit both solid (elastic) and viscous (liquid) char- 
acteristics. The elastic and viscous nature of these materials is described respectively by the storage $\left(G^{\prime}\right)$ and loss $\left(G^{\prime \prime}\right)$ linear moduli. Accordingly, it is possible to quantify the predominance of elastic or viscous character of a sample by submitting it to some external stress while monitoring the above mentioned responses [40-42]. $G^{\prime}$ and $G^{\prime \prime}$ are key parameters for describing the mechanical stability of a system. For instance, how much stress the system can sustain before flowing or how its viscosity changes as the shear rate grows are fundamental for certain applications.

A rheological investigation was conducted aimed at evaluating the mechanical stability of the gel/Cramoll 1-4 immobilizing matrix composed by Cramoll 1-4 mixed into the pure galactomannan gel. The mechanical stability was evaluated by obtaining the viscoelastic properties of the immobilizing gel matrix via rheometry measurements, as a function of the sample storage period, divided into the following steps: (i) A stress-strain experiment where the matrix was submitted to a stress and where the linear moduli $G^{\prime}$ and $G^{\prime \prime}$ were monitored. This measurement starts at a linear reversible regime (small strain) but eventually reaches a nonlinear strain deformation regime where the sample structure fails with subsequent flowing; (ii) a flow curve where the gel/Cramoll 1-4 immobilizing matrix shear stress response was monitored as a function of a strain rate. This is a measurement where the matrix is submitted to a large destructive strain variation as a function of time; (iii) An oscillatory stress study where the gel/Cramoll 1-4 immobilizing matrix was submitted to a low deformation regime ( $0.2 \%$ strain) of oscillations and where the linear elastic (storage), $\mathrm{G}^{\prime}$, and viscous (loss), $\mathrm{G}^{\prime \prime}$, moduli were monitored as a function of a variable oscillatory frequency. In this last experiment, specific structure length scales can be probed non-destructively. The pure galactomannan gel was submitted to the same set of experiments and was used as a control for monitoring the effect of the Cramoll 1-4 upon the immobilizing gel matrix mechanical properties.

3.1.3.1. Stress-strain test. The stress-strain test works as a preliminary investigation where one can separate the linear from non-linear structure response regime as well as identifying the elastic, $G^{\prime}$, and loss, $G^{\prime \prime}$, moduli contributions. The Linear Viscoelastic Region (LVR) is considered as the one encompassing the area where the structure is deformed but not destroyed, represented by the constants $G^{\prime}$ and $G^{\prime \prime}$, independent of stress or strain. This regime indicates the toughness-area under the curve-of the material. On the other hand, the values of $\mathrm{G}^{\prime}$ in this region indicate the stiffness of the material, or the degree of deformation for a given stress.

The results in Fig. 1A and B depict $G^{\prime}$ and $G^{\prime \prime}$ moduli as a function of an applied shear stress for the pure galactomannan gel and the gel/Cramoll 1-4 immobilizing matrix, respectively. To help visualizing the data, we have depicted the 5 and 60 days data as representative of the entire storage period. Nevertheless, on Fig. 1C and $D$, the values of $G^{\prime}$ and $G^{\prime \prime}$, extracted from the LVR from Fig. 1A and B respectively, are presented for the entire storage period recorded.

For the pure galactomannan gel, Fig. $1 \mathrm{~A}$ and $\mathrm{C}$, the results indicate that the gel suffers some mild stiffening-an increase on $\mathrm{G}^{\prime}$-during the storage period. $\mathrm{G}^{\prime}$ and $\mathrm{G}^{\prime \prime}$ are almost identical with $\mathrm{G}^{\prime \prime}>\mathrm{G}^{\prime}$, at Fig. 1A, for the storage period of 5 days. We should mention that $G^{\prime} \gg G^{\prime \prime}$ in a stress-strain test is a rheological signature for a strong elastic gel system. The similarity of $G^{\prime}$ and $G^{\prime \prime}$ values is then indicative of a weak/soft gel behavior. This behavior is in agreement with Albuquerque et al. [20]. For the 60 days storage period, $\mathrm{G}^{\prime}>\mathrm{G}^{\prime \prime}$, reaching though a cross-over, point where $\mathrm{G}^{\prime}=\mathrm{G}^{\prime \prime}=50 \mathrm{~Pa}$, for a stress around $100 \mathrm{~Pa}$. The latter will be considered the stress at which the gel structure fails completely and the system flows. Fig. $1 C$ shows the data, $G^{\prime}$ and $G^{\prime \prime}$ at the LVR, for the entire storage period. Initially, $G^{\prime}$ is approximately equal to $G^{\prime \prime}$ but, as the storage period increases, a reduction of both moduli up to 20 days is observed. This reduction is followed by an increase of both mod- uli with a concomitant separation of their values. A gel stiffening as a function of storage period, for periods longer than 20 days, is indicated by the increase in the difference between $G^{\prime}$ and $G^{\prime \prime}$. We should reinforce that the galactomannan gel behavior depends on concentration.

The moduli-stress curves for the gel/Cramoll 1-4 immobilizing matrix, Fig. 1B and D, indicate that the system suffers both $G^{\prime}$ and $\mathrm{G}^{\prime \prime}$ reduction as a function of the storage period. The data in Fig. 1B, for the storage period of 5 days, is essentially identical to that for the pure galactomannan gel for the same period. However, for the storage period of 60 days, there is a substantial decrease, almost one order of magnitude seen in Fig. 1B, for both moduli. Moreover, the gel/Cramoll 1-4 immobilizing matrix structure for the storage period of 60 days fails at a smaller stress value of approximately $20 \mathrm{~Pa}$. It is important to note a modest maximum for $\mathrm{G}^{\prime \prime}$ at this limit value. This maximum, known as weak strain/stress overshoot, indicates the presence of microstructures that required extra stress in order to align with the flow. The microstructure is not strong enough to cause a similar increase in the elastic modulus, common in shear thickening systems [43]. Fig. 1D shows the entire storage period data. One can notice $G^{\prime}$ approximately equal to $G^{\prime \prime}$ for the entire interval with both moduli decreasing as a function of the storage period. The variation in moduli is more substantial than that observed for the pure galactomannan gel and, moreover, moves in the opposite direction. The gel/Cramoll 1-4 immobilizing matrix shows no variation in its moduli up to 20 days storage period. Nevertheless both moduli reduce substantially for longer periods, the system is said to soften as opposed to the stiffening that occurs in the pure galactomannan gel measurements. The latter is probably due to some aging of the pure gel structure along storage period.

A potential explanation for the decay on $G^{\prime}$ and $G^{\prime \prime}$ moduli for our immobilizing gel matrix comes from Valenga et al. [13] study on the immobilization of the lectin extracted from Concanavalia ensiformis seeds, also known as ConA, onto a monolayer made of a galactomannan extracted from Leucaena leucocephala seeds. They suggested that because the galactomannan backbone is $\beta$-Dmannose $(1 \rightarrow 4)$ linkage and some units at the $C-6$ position carries $\alpha$-D-galactose units, the recognition of ConA might be for $\mathrm{OH}$ groups at the $\mathrm{C}-3$ and $\mathrm{C}-6$ position for the mannose units not substituted. Cramoll 1-4 has its specificity correlated with the ConA, then the interaction between the galactomannan extracted from $C$. grandis and Cramoll 1-4 might also occurs through weak monomeric bindings affinities such as hydrogen bonds and Van der Waals interaction, resulting in some depolymerisation in the central mannose core of the polysaccharide after a period of 60 days. The softening of the gel/Cramoll 1-4 structure as well as the appearance of a microstructure evidenced by the weak shear overshoot might be related with the biding processes described above. Moreover, this biding could also explain a potential degradation of Cramoll 1-4 for long storage periods as will be discussed in Section 3.2.

It is important to mention that, depending on the expected application of the immobilizing gel/Cramoll 1-4 immobilizing matrix, this substantial reduction may not be relevant.

3.1.3.2. Flow curves. The flow curves are experiments where the structure of the sample is tested against rotational flow shear rate and potential yield stresses are predicted. Since we are mostly interested in elastic immobilizing mechanical properties, stress response, where yield stresses can be obtained, instead of apparent viscosity, becomes more informative. Fig. 2 depicts shear stress response of the pure galactomannan gel (2A) and the gel/Cramoll 1-4 immobilizing matrix (2B) as a function of shear rate. The several plots present data for an interval of storage periods ranging from 5 to 90 days for the pure galactomannan gel (2A); and from 5 to 60 days for the gel/Cramoll 1-4 immobilizing matrix (2B). 

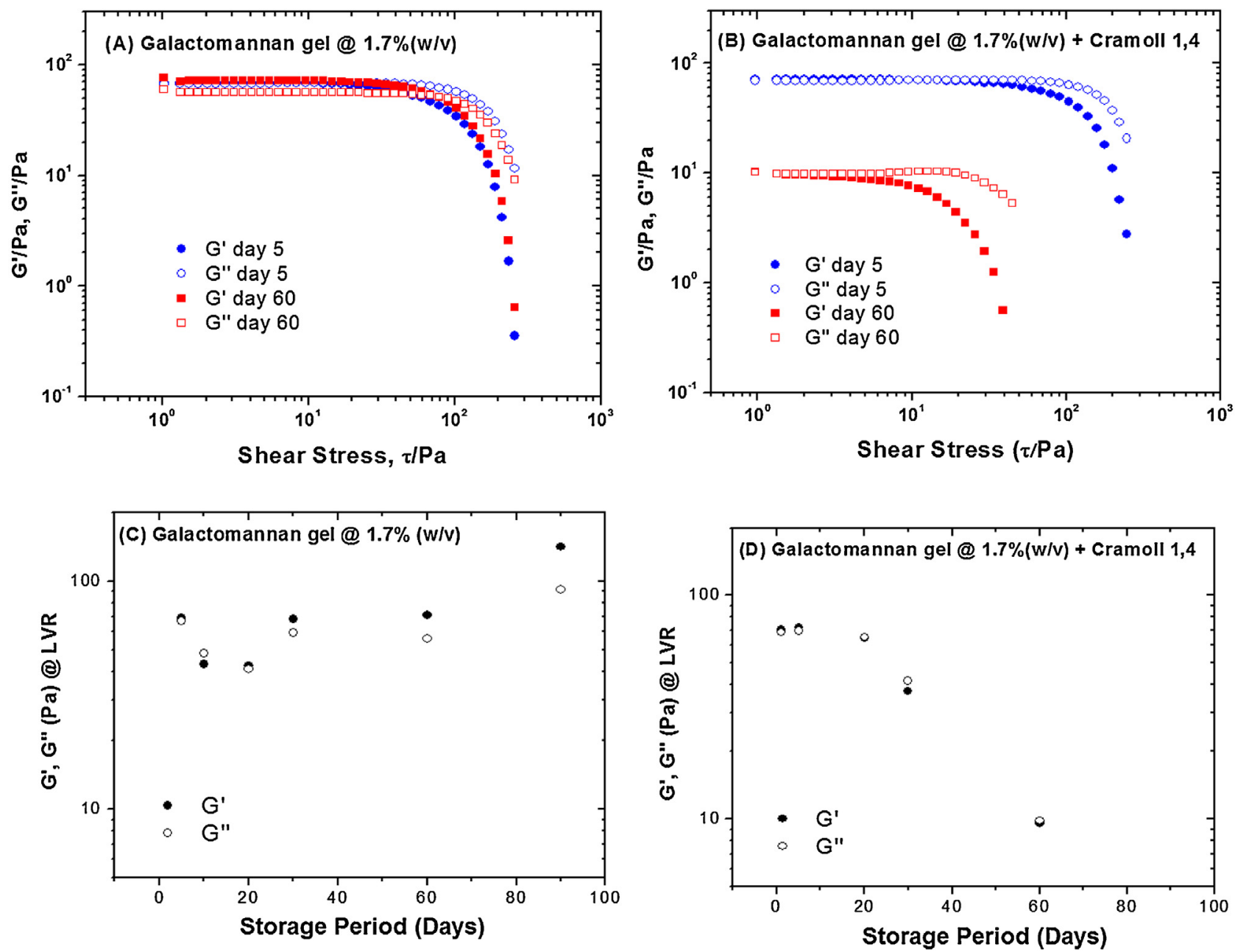

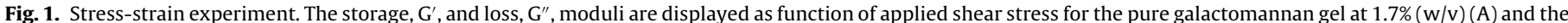


region (LVR) from (A) for several storage periods; (D) the same as (C) for the LVR obtained from (B).
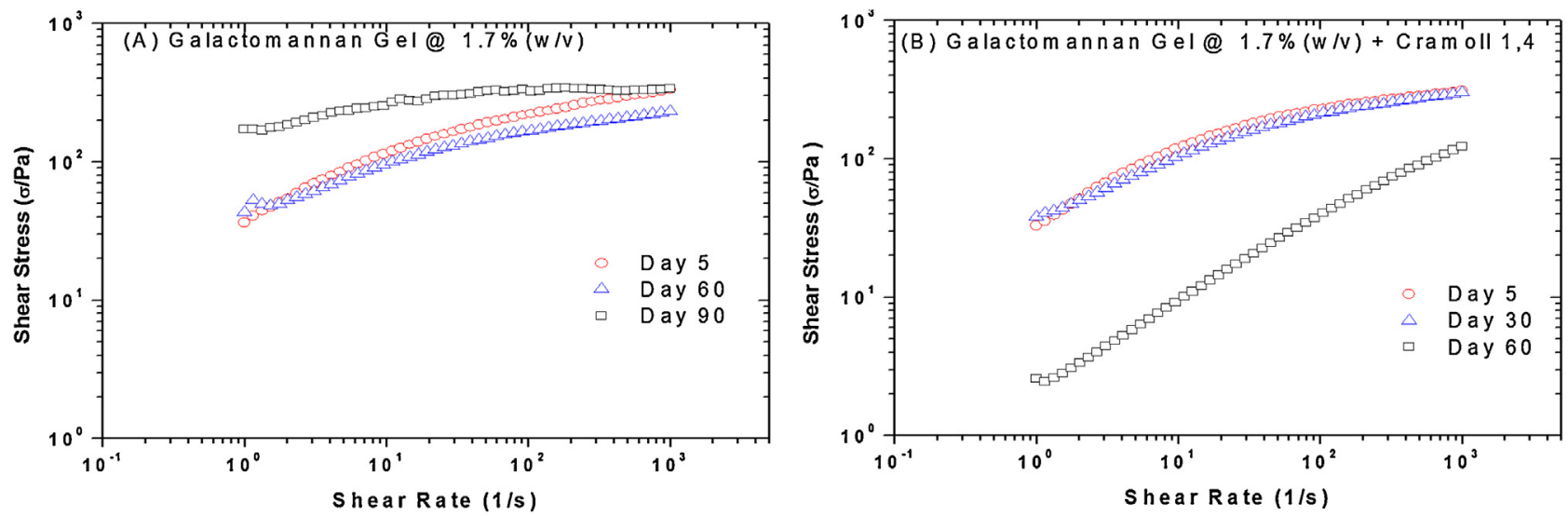

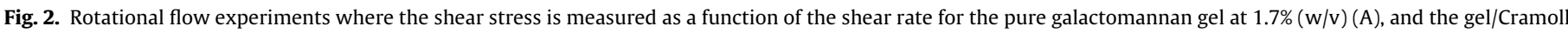
1-4 immobilizing matrix (B) for different storage periods.

The straight forward approach to describe shear stress $\sigma$ as a function of shear rate $\dot{\gamma}$ is via the Herschel-Bulkley (HB) model [44]:

$\sigma=\sigma_{y} k \dot{y}^{n}, \quad$ for $\sigma \geq \sigma_{y}$

where $\sigma_{y}$ denotes the yield stress, and the constant $k$ is the consistency parameter associated with viscosity $\eta=k \dot{y}^{n-1}$ The power $n$ is a fitting parameter of the model describing how non-Newtonian ( $n=1$ for a Newtonian sample) the system is, with $0<n<1$ for shear-thinning, and $n>1$ indicating shear-thickening. Fitting this model to the data on Fig. 2 one is able to obtain both $n$ and the yield stress by extrapolating the experimental data towards zero shear rate. The $n=0.12$ obtained indicate shear-thinning for the pure galactomannan gel identical to that obtained by Albuquerque et al. 
[20]. Shear-thinning is also observed in the gel/Cramoll 1-4 immobilizing matrix but, as the storage period increases, a lower level of shear-thinning, and therefore lower $n$ values are obtained. Overall, for storage periods from 5 to 30 days, both samples behave identically. However, for storage period of 60 days, the matrix exhibits a substantial decrease in the shear stress response, and therefore its apparent viscosity over the entire experimental shear rate interval. This reduction agrees with the stress-strain response already discussed. The extrapolated yield stress for the latter storage period is really small and can be neglected. The shear-thinning reduction, indicated by the value of $n=0.3$, gives evidence to a reduction in the matrix structure length scale. One should consider at this point, from the rheology perspective, the gel/Cramoll 1-4 immobilizing matrix as a viscoelastic fluid.

The shear thinning behavior observed for the pure galactomannan gel (Fig. 2A) is similar to that reported for others galactomannans, such as those extracted from $L$. leucocephala seeds [45] and the most commercially used galactomannan, guar gum [46]. It is important to note though that this behavior is dependent on the molecular weight [5] the mannose/galactose ratio [19] and galactomannan concentration [18]. Moreover, the presence of Cramoll 1-4 appears not to be relevant regarding the degree of shear-thinning since both samples behave similarly within a given storage period from 5 to 30 days. Nevertheless, the pure galactomannan sample shows an increase in the stress response, as well as an increase in the yield stress, for the 90 days storage period. This is the same stiffening effect observed for the stress-strain measurements.

The gel/Cramoll 1-4 immobilizing matrix (2B), also kept under the same controlled storage conditions, presented similar stress response up to 30 days, decreasing its value significantly for 60 days storage period. The yield stress, obtained from the extrapolated theory fit, is negligible, again, for the latter storage period. This null yield stress agrees with the viscoelastic fluid signature observed by the stress-strain test.

3.1.3.3. Oscillatory results. Fig. 3 shows the results of $G^{\prime}$ and $G^{\prime \prime}$ moduli of the pure galactomannan gel $(3 \mathrm{~A})$ and the gel/Cramoll 1-4 immobilizing matrix (3B) as a function of oscillatory frequency for a small amplitude oscillatory strain. The experiments were conducted keeping the samples at the LVR, where the gel structure is deformed reversibly and not destroyed, offering the opportunity to probe the microstructure of both systems as a function of the experimental storage period.

At the reversible small strain regime, the addition of Cramoll 1-4 on the pure galactomannan gel did not affect substantially, apart from a mild global reduction in both moduli, the rheological signature of a weak gel which consist of a small variation of $\mathrm{G}^{\prime}$ and $\mathrm{G}^{\prime \prime}$ as a function of frequency. The gel signature also agrees with visual observation of the samples standing against gravity. One can observe that both moduli continuously increase as a function of frequency for the storage interval depicted.

The cross-over between $G^{\prime}$ and $G^{\prime \prime}$ for both samples up to 30 days indicated a fluid behavior for small oscillation frequencies, dissipating most of the externally applied energy $\left(G^{\prime \prime}>G^{\prime}\right)$, similar to the results reported by Albuquerque et al. [20] for the galactomannan extracted from $C$. grandis seeds at approximately $1.5 \%$ $(\mathrm{w} / \mathrm{v})$ and also to the oscillatory behavior observed for other galactomannans $[45,47]$. There was observed an inversion $\left(G^{\prime \prime}<G^{\prime}\right)$ in the range between 3 and $9 \mathrm{~Hz}$ frequency, and the pure galactomannan gel acquired an elastic behavior (3A). The crossover disappears for the 60th day, so the two moduli evolved almost parallel with frequency increase; for the 90th day, the system had reached a gel state determined by an enhancement of $\mathrm{G}^{\prime}$ and $\mathrm{G}^{\prime \prime}$ values (data not shown).
The classical gel behavior $\left(G^{\prime}\right.$ parallel to $G^{\prime \prime}$ as a function of frequency) was observed by Soares et al. [6] for the hydrogel composed by $\kappa$-carrageenan and the pure galactomannan gel. The pure galactomannan hydrogel was rheologicaly tested during 90 days period and a stiffening process was observed where both $\mathrm{G}^{\prime}$ and $\mathrm{G}^{\prime \prime}$ increase as a function of storage. An additional rheological study of the pure galactomannan gel as function of storage period may offer new opportunities for preparing solutions with smaller galactomannan concentrations that can exhibit a gel state after stiffening for a certain storage period.

\subsection{Biological Activity of free Cramoll 1-4 and gel/Cramoll 1-4 immobilizing matrix}

A range of Cramoll 1-4 concentrations was already evaluated: Maciel et al. [29] investigated Cramoll 1-4 in the mitogenic stimulation of human lymphocytes varying the lectin concentration from 0.78 to $100 \mu \mathrm{g} / \mathrm{ml}$; Melo et al. [32] examined, in vivo, clinical and histopathological aspects of cutaneous wounds performed experimentally in healthy and immunocompromised mice using $100 \mu \mathrm{g} / \mathrm{ml}$ of Cramoll 1-4; Avelino et al. [48] described the development of a biosensor composed by $200 \mu \mathrm{g} / \mathrm{ml}$ Cramoll 1-4 immobilized by electrostatic interactions on hybrid Nanocomposite (gold nanoparticles and polyaniline) to distinguish abnormal glycoproteins of sera from patients infected with dengue serotypes I, II and III. Considering the broad range of Cramoll 1-4 concentrations in the development of their biological activities, a medium value of $100 \mu \mathrm{g} / \mathrm{ml}$ was chosen to be immobilized into the pure galactomannan gel.

The HA comparisons were made to a Cramoll 1-4 free solution which was benchmarked to $100 \%$ HA during the entire period. The HA of Cramoll 1-4 free solution was 3.01. Gel/Cramoll 1-4 immobilizing matrix presented HA of 2.71 when performed on days 1, 5 and 20 , while for 30 and 60 days, its HA reduced to 1.81 . HA was not significant after 90 days. These results demonstrate that the immobilized Cramoll 1-4 retained 90\% of its initial HA during 20 days, decreasing to $60 \%$ up to 60 days.

The fundamental characteristic of lectins is its ability to bind specifically with carbohydrates, for instance, with glycoconjugates on cell surface. When that occurs, lectins deploy several biological effects. The HA allows for determining the lectin presence in a sample by forming a network agglutination between lectin binding sites and carbohydrates of the erythrocytes surface [49].The HA stability of the gel/Cramoll 1-4 immobilizing matrix ensures the maintenance of all lectin biological activities up to 60 days.

Cramoll 1-4 is classified as part of the mannose/glucose-specific binding group, as these are the monosaccharides to which Cramoll 1-4 exhibits the highest binding affinity. It is reasonable to predict that specific and non-specific hydrogen interactions between gel and lectin positively charged residues might occur, since the pure galactomannan gel has a central core of $\alpha(1 \rightarrow 4)$-linked Dmannopyranose to which $\beta(1 \rightarrow 6)$-linked $\alpha$-D-galactopyranosyl units are attached. That could be explained by potential conformational changes of Cramoll 1-4 for long storage periods.

The decrease in the opacity value for the gel/Cramoll 1-4 immobilizing matrix over time is, as already indicated in the flow curves, associated with the loss of gel structure stability. The stability loss seems to be corroborated by the loss of activity in HA during the same period, which might be associated with specific binding of the Cramoll 1-4 with the galactomannan backbone.

\subsection{Microbial evaluation}

A spoiled product may be described as one that has been rendered unfit for use. Microbial spoilage can be caused by bacteria, yeasts or fungi, which are all extremely versatile in their 

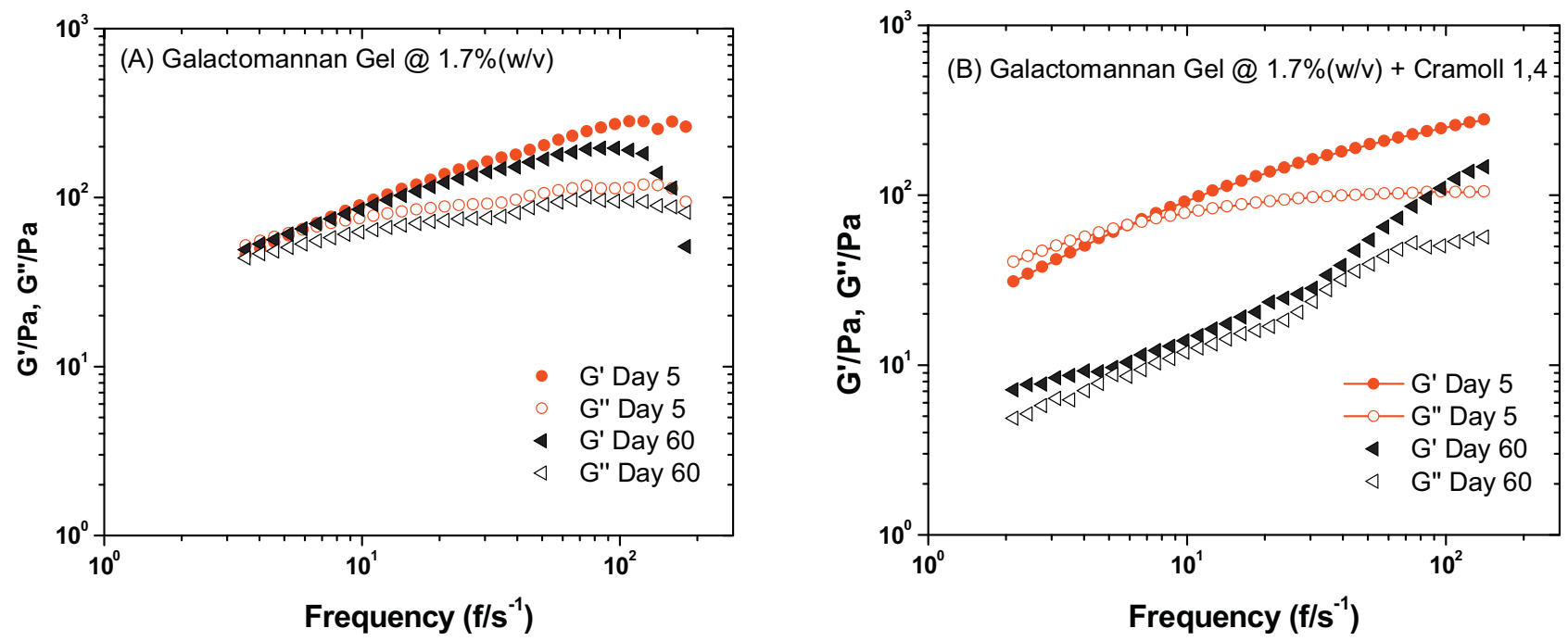

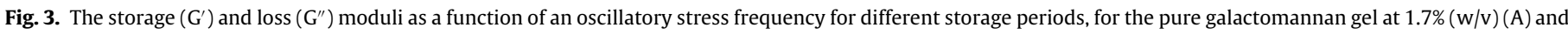
the gel/Cramoll 1-4 immobilizing matrix (B).

metabolic activities. All classes of natural organic compounds, such as polysaccharides and proteins, are susceptible to degradation by the above mentioned microorganisms; synthetic compounds are also attacked, although often less readily [50]. To prevent the negative consequences ofmicrobial contamination, careful control measures are required during the storage process for industrial products, even food or cosmetics.

The pure galactomannan gel and the gel/Cramoll 1-4 immobilizing matrix presented no microbial counting for cultivable microorganisms along the total experimental interval. Our results are in accordance with the microbiological analysis of Soares et al. [6] for the hydrogel composed by к-carrageenan and the pure galactomannan gel, since there was no observed microorganism growth in any of the experimentation days.

\section{Conclusion}

The gel/Cramoll 1-4 immobilizing matrix, as confirmed by the rheological measurements, proved to be stable up to 30 days with substantial variations occurring after this period. Moreover, throughout the entire storage period, no microbial contamination occurred, no changes in the neutral $\mathrm{pH}$ of the gels were observed and the colorimetric parameters remained constant. $90 \%$ of the HA was observed after 20 day of storage, decreasing to $60 \%$ up to 60 days. The rheological properties of the/Cramoll 1-4 immobilizing matrix and the maintenance of the HA of the immobilized Cramoll for a long period of time indicate the suitability for its use in topical applications, with potential use in pharmaceutical and cosmetics industries.

\section{Acknowledgments}

Priscilla B.S. Albuquerque is a recipient of a scholarship from the Fundação de Amparo à Ciência e Tecnologia do Estado de Pernambuco (FACEPE) and Paulo A. G. Soares is a recipient from the Coordenação de Aperfeiçoamento de Pessoal de Nível Superior (CAPES). The authors express their gratitude to the Conselho Nacional de Desenvolvimento Científico e Tecnológico (CNPq) for research grants and fellowships (MTSC, LCBBC and MGCC). The authors acknowledge the Centro de Tecnologias Estratégicas do Nordeste (CETENE) for analytical support and CNPq as well as CAPES for financial support.

\section{References}

[1] L. Yang, L. Zhang, Chemical structural and chain conformational characterization of some bioactive polysaccharides isolated from natural sources, Carbohydr. Polym. 76 (2009) 349-361.

[2] C.W. Vendruscolo, C. Ferrero, E.A.G. Pineda, J.L.M. Silveira, R.A. Freitas, M.R. Jimenez-Castellanos, T.M.B. Bresolin, Physicochemical and mechanical characterization of galactomannan from Mimosa scabrella: effect of drying method, Carbohydr. Polym. 76 (2009) 86-93.

[3] M.A. Pollard, B. Eder, P. Fischer, E.J. Windhab, Characterization of galactomannans isolated from legume endosperms of Caesalpinioideae and Faboideae subfamilies by multidetection aqueous SEC, Carbohydr. Polym. 79 (2010) 70-84

[4] H. Neukom, Galactomannans: Properties and Applications, 22, Lebensmmitel Wissenschaft und Technologie, London, 1989.

[5] A.I. Bourbon, A.C. Pinheiro, C. Ribeiro, C. Miranda, J.M. Maia, J.A. Teixeira, A.A. Vicente, Characterization of galactomannans extracted from seeds of Gleditsia triacanthos and Sophora japonica through shear and extensional rheology: comparison with guar gum and locust bean gum, Food Hydrocoll. 24 (2010) $184-192$.

[6] P.A.G. Soares, J.R.P.C. Seixas, P.B.S. Albuquerque, G.R.C. Santos, P.A.S. Mourão, W. Barros Júnior, M.T.S. Correia, M.G. Carneiro-da-Cunha, Development and characterization of a new hydrogel based on galactomannan and K-carrageenan, Carbohydr. Polym. 134 (2015) 673-679.

[7] M.A. Cerqueira, B.W.S. Souza, J.T. Martins, J.A. Teixeira, A.A. Vicente, Seed extracts of Gleditsia triacanthos: functional properties evaluation and incorporation into galactomannan films, Food Res. Int. 43 (2010) 2031-2038.

[8] M.A. Cerqueira, M.J. Sousa-Gallagher, I. Macedo, R. Rodriguez-Aguilera, B.W.S Souza, J.A. Teixeira, A.A. Vicente, Use of galactomannan edible coating application and storage temperature for prolonging shelf-life of "Regional" cheese, J. Food Eng. 97 (2010) 87-94.

[9] J.T. Martins, M.A. Cerqueira, A.I. Bourbon, A.C. Pinheiro, B.W.S. Souza, A.A. Vicente, Synergistic effects between $\kappa$-carrageenan and locust bean gum on physicochemical properties of edible films made thereof, Food Hydrocoll. 29 (2012) 280-289.

[10] L. Gilbert, V. Loisel, G. Savary, M. Grisel, C. Picard, Stretching properties of xanthan carob, modified guar and celluloses in cosmetic emulsions, Carbohydr. Polym. 93 (2013) 644-650.

[11] R. Alvarez-Román, G. Barré, R.H. Guy, H. Fessi, Biodegradable polymer nanocapsules containing a sunscreen agent: preparation and photoprotection, Eur. J. Pharm. Biopharm. 52 (2001) 191-195.

[12] S. Comba, R. Sethi, Stabilization of highly concentrated suspensions of iron nanoparticles using shear-thinning gels of xanthan gum, Water Res. 43 (2009) 3717-3726.

[13] F. Valenga, D.F.S. Petri, N. Lucyszyn, T.A. Jó, M.R. Sierakowski, Galactomannan thin films as supports for the immobilization of Concanavalin A and/or dengue viruses, Int. J. Biol. Macromol. 50 (2012) 88-94.

[14] C.W. Vendruscolo, I.F. Andreazza, J.L.M.S. Ganter, C. Ferrero, T.M.B. Bresolin, Xanthan and galactomannan (from M. scabrella) matrix tablets for oral controlled delivery of theophylline, Int. J. Pharm. 296 (2005) 1-11.

[15] Y. Wu, W. Cui, N.A.M. Eskin, H.D. Goff, An investigation of four commercial galactomannans on their emulsion and rheological properties, Food Res. Int. 42 (2009) 1141-1146. 
[16] P.B. Fernandes, M.P. Gonçalves, J.L. Doublier, A rheological characterization of Kappa-carrageenan/galactomannan mixed gels: a comparison of locust bean gum samples, Carbohydr. Polym. 16 (1991) 253-274.

[17] M.M. Cross, Rheology of non-Newtonian fluids: a new flow equation for pseudoplastic systems, J. Coll. Interface Sci. 20 (1965) 417.

[18] N. Garti, Z. Madar, A. Aserin, B. Sternheim, Fenugreek galactomannans as food emulsifiers, Food Sci. Technol. (Leb.) 30 (1997) 305-311.

[19] W. Sittikijyothin, D. Torres, M.P. Gonçalves, Modelling the rheological behaviour of galactomannan aqueous solutions, Carbohydr. Polym. 59 (2005) 339-350.

[20] P.B.S. Albuquerque, W. Barros Jr., G.R.C. Santos, M.T.S. Correia, P.A.S. Mourão, J.A. Teixeira, M.G. Carneiro-da-Cunha, Characterization and rheological study of the galactomannan extracted from seeds of Cassia grandis, Carbohydr. Polym. 104 (2014) 127-134

[21] N. Sharon, H. Lis, The structural basis for carbohydrate recognition by lectins, in: A.M. Wu (Ed.), The Molecular Immunology of Complex Carbohydrates-2, Kuwer Academic/Plenum Publishers, Tawain, 2001.

[22] M.T.S. Correia, L.C.B.B. Coelho, Purification of a glucose/mannose specific lectin isoform 1, from seeds of Cratylia mollis mart (Camaratu Bean), Appl. Biochem. Biotech. 55 (1995) 261-273.

[23] P.M.G. Paiva, L.C.B.B. Coelho, Purification and partial characterization of two lectinisoforms from Cratylia mollis Mart. (Camaratu Bean), Appl. Biochem. Biotechnol. 36 (1992) 113-118.

[24] C.A.S. Andrade, M.T.S. Correia, L.C.B.B. Coelho, S.C. Nascimento, N.S. Santos-Magalhães, Antitumor activity of Cratylia mollis lectin encapsulated into liposomes, Int. J. Pharm. 278 (2004) 435-445.

[25] M.P. Fernandes, N.M. Inada, M.R. Chiaratti, F.F.B. Araújo, F.V. Meirelles, M.T.S Correia, L.C.B.B. Coelho, M.J.M. Alves, F.R. Gadelha, A.E. Vercesi, Mechanism of Trypanosoma cruzi death induced by Cratylia mollis seed lectin, J. Bioenerg. Biomembr. 42 (2010) 69-78.

[26] C.M.L. Melo, A.L.R. Lima, E.I.C. Beltrão, C.C.B. Cavalcanti, M.R. Melo-Júnior S.M.L. Montenegro, L.C.B.B. Coelho, M.T.S. Correia, A.M.A. Carneiro-Leão, Potential effects of Cramoll 1 lectin on murine Schistosomiasis mansoni, Acta Trop. 118 (2011) 152-158.

[27] C.M.L. Melo, M.C.A.B. Castro, A.P. Oliveira, F.O.S. Gomes, V.R.A. Pereira, M.T.S. Correia, L.C.B.B. Coelho, P.M.G. Paiva, Immunomodulatory response of Cramoll 1-4 lectin on experimental lymphocytes, Phytother Res. 24 (2010) 1631-1636.

[28] C.M.L. Melo, B.A. Paim, K.G. Zecchin, J. Morari, M.R. Chiarrati, M.T.S. Correia, L.C.B.B. Coelho, P.M.G. Paiva, Cramoll 1-4 lectin increases ROS production, calcium levels and cytokine expression in treated spleen cells of rats, Mol. Cell. Biochem. 342 (2010) 163-169.

[29] E.V.M. Maciel, V.S. Araújo-Filho, M. Nakazawa, Y.M. Gomes, L.C.B.B. Coelho, M.T.S. Correia, Mitogenic activity of Cratylia mollis lectin on human lymphocytes, Biology 32 (2004) 57-60.

[30] C.M.L. Melo, H. Melo, M.T.S. Correia, L.C.B.B. Coelho, M.B. Silva, V.R.A. Pereira, Mitogenic response and cytokine production induced by cramoll 1-4 lectin in splenocytes of inoculated mice, Scand. J. Immunol. 73 (2011) 112-121.

[31] V.L.M. Lima, M.T.S. Correia, Y.M.N. Cechinel, C.A.M. Sampaio, J.S. Owenand, L.C.B.B. Coelho, Immobilized Cratylia mollislectin as a potential matrix to isolate plasma glycoproteins, including lecithin-cholesterol acyltransferase, Carbohydr. Polym. 33 (1997) 27-32

[32] C.M.L. Melo, C.S. Porto, M.R. Melo-Júnior, C.M. Mendes, C.C.B. Cavalcanti, L.C.B.B. Coelho, A.L.F. Porto, A.M.A. Carneiro-Leão, M.T.S. Correia, Healing activity induced by Cramolll 1-4 lectin in healthy and immunocompromised mice, Int J. Pharm. 408 (2011) 113-119.
[33] D.H. Bing J.G.M. Weyand, A.B. Stavitsky, Hemagglutination with aldehyde-fixed erythrocytes for assay of antigens and antibodies, Proc. Soc. Exp. Biol. Med. 124 (1967) 1166-1170.

[34] C. Vanderzant, D.F. Splittstoesser, Compendium of Methods for the Microbiological Examination of Foods, third ed., American Public Health Association (APHA), Washington, 1992.

[35] M. Srivastava, V.P. Kapoor, Seed galactomannans: an overview, Chem. Biodivers. 2 (2005) 295-317.

[36] T. Toniazzo, I.F. Berbel, S. Cho, C.S. Favaro-Trindade, I.C.F. Moraes, S.C. Pinho, b-Carotene-loaded liposome dispersions stabilized with xanthan and guar gums: physico-chemical stability and feasibility of application in yogurt, LWT-Food Sci. Technol. 59 (2014) 1265-1273.

[37] J.H. Segura, F.B. Camargo Jr., E. Bagatin, M.B.G.M. Campos, Influence of thermal water and its oligoelements in the stability and efficacy of dermocosmetics formulations, Surg. Cosmt. Dermatol. 2 (2010) 11-17.

[38] M.R. Martelli, T.T. Barros, O.B.G. Assis, Puree films from bananas processed in the batching mode: mechanical properties and coloring variations, Polímeros 24 (2014) 137-142.

[39] B. Li, J.F. Kennedy, J.L. Peng, X. Yie, B.J. Xie, Preparation and performance evaluation of glucomannan-chitosan-nisin ternary antimicrobial blend film, Carbohydr. Polym. 65 (2006) 488-494.

[40] F. Iagher, F. Reicher, J.L.M.S. Ganter, Structural and rheological properties of polysaccharides from mango (Mangifera indica L.) pulp, Int. J. Biol. Macromol. 31 (2002) 9-17.

[41] G.M. Kavanagh, S.B. Ross-Murphy, Rheological characterization of polymer gels, Prog. Polym. Sci. 23 (1998) 533-562.

[42] G.E. Perissutti, T.M.B. Bresolin, J.L.M.S. Ganter, Interaction between the galactomannan from Mimosa scabrella and milk proteins, Food Hydrocoll. 16 (2002) 403-417.

[43] K. Hyun, S.H. Kim, K.H. Ahn, S.J. Lee, Large amplitude oscillatory shear as a way to classify the complex fluids, J. Non-Newtonian Fluid Mech. 107 (2002) 51-65.

[44] H.A. Barnes, Thixotropy-a review, J. Non-Newtonian Fluid Mech. 70 (1997) $1-33$.

[45] L.M. Nwokocha, P.A. Williams, Rheological characterization of the galactomannan from Leucaena leucocephala seed, Carbohydr. Polym. 90 (2012) 833-838.

[46] D. Mudgil, S. Barak, B.S. Khatkar, Effect of enzymatic depolymerization on physicochemical and rheological properties of guar gum, Carbohydr. Polym. 90 (2012) 224-228

[47] M.J. Perduca, M.J. Spotti, L.G. Santiago, M.A. Judis, A.C. Rubiolo, C.R. Carrara, Rheological characterization of the hydrocolloid from Gleditsia amorphoides seeds, LWT-Food Sci. Technol. 51 (2013) 143-147.

[48] K.Y.P.S. Avelino, C.A.S. Andrade, C.P. Melo, M.L. Nogueira, M.T.S. Correia, L.C.B.B. Coelho, Biosensor based on hybrid nanocomposite and Cramoll lectin for detection of dengue glycoproteins in real samples, Synth. Met. 194 (2014) $102-108$.

[49] M.T.S. Correia, L.C.B.B. Coelho, P.M.G. Paiva, Lectins, carbohydrate recognition molecules: are they toxic? in: Y.H. Siddique (Ed.), Recent Trends in Toxicology, Transworld Research Network, India, 2008, pp. 47-59.

[50] R. Smart, D.F. Spooner, Microbiological spoilage in pharmaceuticals and cosmetics J. Soc. Cosmetic Chem. 23 (1972) 721-737. 